\title{
ENERGY TRANSFER. TO ELECTRONS IN FAST PROTON COLLISIONS
}

L. H. Toburen and W. A. Glass

Battelle Memorial Institute

Pacific Northwest Laboratory

Richland, Washington 99352

Apri1 28, 1971

Paper for oral presentation at the annual meeting of the Radiation Research Society, 9-13 May 71, Boston, Massachusetts.

This paper is based on work performed under United States Atomic Energy Commission Contract AT(45-1)-1830.

This report was prepared as an account of work sponsored by the United States Government. Neither the United States nor the United Stales Alumi Encrgy Commission, nor any of their employees, nor anyees, makes any warranty, express or implied, or assumes any legal liability or responsibility for the accuracy, completeness or usefulness of any information, apparatus, preteness or useclosed, or represents that its use would not infringe privately owned rights. 


\section{DISCLAIMER}

This report was prepared as an account of work sponsored by an agency of the United States Government. Neither the United States Government nor any agency Thereof, nor any of their employees, makes any warranty, express or implied, or assumes any legal liability or responsibility for the accuracy, completeness, or usefulness of any information, apparatus, product, or process disclosed, or represents that its use would not infringe privately owned rights. Reference herein to any specific commercial product, process, or service by trade name, trademark, manufacturer, or otherwise does not necessarily constitute or imply its endorsement, recommendation, or favoring by the United States Government or any agency thereof. The views and opinions of authors expressed herein do not necessarily state or reflect those of the United States Government or any agency thereof. 


\section{DISCLAIMER}

Portions of this document may be illegible in electronic image products. Images are produced from the best available original document. 


\section{ENERGY TRANSFER TO ELECTRONS IN FAST PROTON COLLISIONS*}

\section{H. Toburen and W. A. Glass \\ Battelle Memorial Institute \\ Pacific Northwest Laboratory \\ Richland, Washington. 99352}

Various physical models for the action of ionizing radiation on chemical or biological systems have one thing in common: They all assume a causative relation between the initial distribution of energy and the final observable product. In such models, whether based on average behavior of the radiation or on its stochastic properties, the track of the charged particle is fundamental to their development. In the sensc used here, the term track is meant to refer to the initial spatial distribution of ionizing interactions initiated by the passage of a charged particle. For heavy charged particles, the cross sections for the production of secondary electrons through primary collibiuns and tho energy and angular distributions of these secondaries are fundamental to track structure. The fragmentary nature of information regarding these primary collisions has prompted our study of the energy and angular distributions of electrons ejected by fast proton collisions in thin gas targets. These results then become the source term for calculations concerning track structure. The nature of the calculations will depend upon whether one is dealing with stochastic models or with models involving mean ionization distributions.

* This paper is based on work performed under United States Atomic Energy Commissiun Controct AT $(45-1)-1830$. 
The particular reaction which we have studied is illustrated in Fig. I along with a schematic drawing of the apparatus. The experiment consists of measuring the energy and angle of the outgoing electron as a function of the incident proton energy. The ejected electrons, commonly referred to as delta-rays, are detected for ejection angles from 20 to 130 degrees with respect to the proton beam. These angular limits were set by the physical size and arrangement of the instrumentation. A differentially pumped cell maintained the target gas at a pressure low enough that the probability of a collision occurring was about 1 in 100 . This low pressure assures that the electrons which were observed resulted from single collisions and also minimizes scatter of the electron in the target. The proton beam was collimated by a multi-aperture arrangement, which reduced the number of background electrons produced in the slit edges. After the beam had passed through the target gas, it was collected by a shielded Faraday cup, the output of which was used in determining absolute values for the electron ejection cross sections. Energy spectra for the ejected electrons was obtained by using a cylindrical mirror electrostatic analyzer collimated to an acceptance angle of approximately 5 degrees. By moving the analyzer, it was possible to change the angle of incidence at which electrons were analyzed. Individual electrons of known energy and angle were detected and counted for a given number of protons passing through the target cell. After corrections for scatlering of very-low-energy electrons in the target cell, absolute values for the cross section were determined. 
A typical example of the cross sections measured in this work is shown in Fig. 2. These results were obtained for $1.0 \mathrm{MeV}$ protons on molecular hydrogen. Eleçtron spectra (cross sections) are shown at representative angles from near the forward direction to 130 degrees in the backward direction. A peak in the cross section is observed at energies corresponding to the kinematic maximum for energy transfer to a free electron. A free electron model would predict a $\delta$-function distribution instead of the broad peaks observed in the measured spectra. The energy of this maximum decreases as the angle increases and is not observed at angles greater than 90 degrees. The emission cross sections also show an increase in value for low-energy electrons. This increase is due to the ejection of electrons by resonant and/or distant collisions. The cross. sections for such collisions are more nearly isotropic than are those for ejection of the higher energy knock-on electrons. For higher atomic number, these low-energy interactions increase in magnitude and the angular dependence of the cross section decreases.

In considering track structure or energy deposition, one is interested not only in the probability of ejccting electrons with a particular energy spectrum, but also in the amount of energy transferred by these electrons. For example, a $2 \mathrm{keV}$ electron will transfer and deposit a large portion of its energy some distance from the original path of the proton, whereas low-energy electrons are assumed to deposit all of there energy locally. We have, therefore, calculated cross sections for energy transfer as a function of the electron energy and angle of emission. Such quantities are displayed in Fig. 3 for $1.5 \mathrm{MeV}$ protons on hydrogen. 
The radial vector represents the total energy per unit solid angle transferred to secondary electrons in a given direction with respect to the proton beam. This quantity is obtained by integrating the energy transfer cross sections with respect to the electron energy according to Equation (1).

$$
\sigma_{\varepsilon}=\int_{\varepsilon_{1}}^{\infty} \varepsilon \sigma(\varepsilon, \theta) d \varepsilon
$$

Three lower limits of integration have been set to illustrate the affects on the distribution of energy transfer caused by excluding short range secondary electrons. The outer curve in Fig. 3 represents the total transfer of energy as a function of angle. The curve obtained using a lower limit of $50 \mathrm{eV}$ indicates the change introduced by excluding all electrons with energies less than the mean energy for all secondaries. A lower limit of. $200 \mathrm{eV}$ was chosen to exclude all electrons which are generally considered to be locally absorbed along the pruluin track. It is observed that a large portion of the cross section for energy transfer in the forward direction is associated with electrons greater than $200 \mathrm{eV}$ while practically no energy is transferred into angles greater than 90 degrees by these electrons.

Quantitative information concerning the fraction of the total energy which is initially transferred into a given direction can be obtained by integrating these energy transfer cross sections with respect to the solid angle. For a given angular interval, the solid angle subtended by a cone in the forward or backward direction is small compared to that 
subtended at angles near 90 degrees. Hence, the fraction of the total energy going into small angles usually will be small even though the cross section for energy transfer is large in forward direction. The result of integrating over the solid angle is illustrated for the case of hydrogen in Fig. 4. The upper portion of the figure displays the same information as was shown in Fig. 3, however, the cosine scale permits a more direct comparison of the total energy transfer in any direction; the area under the curve between two selected angles represents energy transferred into that segment of solid angle. The histogram displays the percentage of the total energy which is transferred into cones of equal angular separation. For hydrogen bombarded with $1.5 \mathrm{MeV}$ protons, the greatest fraction of the energy is transferred into a cone between the angles of 60 and 70 degrees. In general, the peak in distributions moves toward smaller angles as the electron energies considered are increased, i.e., as the lower limit of the energy integral is increased. At the smaller angles, the largest fraction of the energy is transferred to electrons with energies greater than $200 \mathrm{eV}$.

The effect of increasing the complexity of the target molecule is evident from the results for protons on nitrogen shown in Fig. $=5$. These results illustrate the effects of the more tightly bound electrons on the angular distribution of energy transfer. The peak observed at about 60 degrees in the upper portion of the figure is attributed to energy losses by the proton to loosely bound electrons; this maximum is at approximately the same angle as for electrons ejected from hydrogen. The second peak, which occurs at somewhat smaller angles, is the result of interactions with 
the tightly bound K-shell electrons. These interactions necessarily involve larger energy transfer which require small impact parameters and hence the electron ejection is in a more forward direction. As is indicated in . the histogram, the interaction of protons with more complex molecules yields a wider spread in the angular distribution of energy transfer. In nitrogen, some energy is transferred in the backward direction by electrons having energy greater than $200 \mathrm{ev}$; such a transfer was not present for hydrogen. These electrons are due primarily to Auger transitions which arise from vacancies in the inner atomic shell. Each such transition results in an electron of approximately $365 \mathrm{eV}$ and leaves the residual target doubly ionized. This energetic electron is in addition to the knockon electron produced by the primary ionization.

The relative contribution of Auger electrons produced in nitrogen by protons of varying energies is illustrated in Fig. 6. The fraction of the total energy which can be attributed to Auger electrons approaches 5 percent for protons of $1 \mathrm{MeV}$ and increases only slightly as the proton energy is further increased. As the atomic number is increased, the numher of. electrons which can become involved in Auger processes is greater and one observes that Auger electrons account for an increasing fraction of the total energy. This increase will be modified at higher $z$ as the competing process of $X$-ray fluorescence becomes more important. Such an increase in the Auger contribution is qual itatively apparent' in Fig. 7, which shows the results of $2 \mathrm{MeV}$ protons on xenon. For instance, there is a much larger fraction of the energy which appears at angles greater than 90 .degrees with respect to the forward direction of the proton beam 
than was found in the case of nitrogen; Auger. electrons contribute a large fraction of this energy. One also notes that the distribution of energy as displayed in the histogram is much broader than was the case for less complex atoms. The energy transfer cross section data displayed in the upper portion of Fig. 7 shows three peaks which can be attributed to the three atomic shells (M, N, and 0 ), which can be excited by a 2 MeV proton. Electrons more tightly bound do not interact inelastically to produce knock-on electrons with protons of this energy.

The angular dependence of the energy transfer to electrons is a strong function of the incident proton energy. This dependence is shown in Fig. 8 where the energy transfer cross sections for both hydrogen and nitrogen are shown for two different proton energies. At the lower incident energies, the relative number of ejected electrons having energies near the binding energy is larger. The net effect of this binding is to cause the distributions to peak in the forward direction. At higher proton energies, the effects of electronic binding became less evident, particularly for the more energetic secondaries ejected in the forward direction. For these energies, the general shape of the measured energy transfer distributions approaches that calculated assuming unbound electrons as shown by the dashed line in Fig. 8. This is most evident in the case of hydrogen where all of the electrons are loosely bound. The infinite probability of energy transfer at 90 degrees predicted by the free electron model is the result of a very large number of distant collisions each of which transfers only a small amount of energy. These distant collisions do not directly result in ejected electrons since the energy 
transferred is small compared to the binding energy. Hence, the finite values for the measured cross sections. Note that the contribution to energy transferred in the backward direction must be totally accounted for by effects of binding. For molecules where inner electronic shells exist, electrons ejected from the outer shells result in energy transfer distributions similar to those from hydrogen, whereas, the more tightly bound electrons contribute to energy transfer in a more forward direction. This is evident in the two maxima which occur in the energy transfer. curves for $1.7 \mathrm{MeV}$ protons on nitrogen which are shown in Fig. 8 . The more forward peak is associated with electrons from the K-shell. Electron ejection from the outer shel is of nitrogen peak at nearly the same angle as for hydrogen. The effects of inner shell ionization in nitrogen are apparent even at .3 MeV as an enhancement of the cross section at small angles not present in the case of hydrogen.

From these data, it is evident that the free electron model is inadequate even as a snurse term for a realistic development of track structure models. Calculations based on the Born approximation and on a binary encounter model produce total ionization cross sections and'energy distributions which are in reasonable agreement with these experimental results. Such calculations, however, are limited to hydrogenic approximations and are severely limited at energies below a few MeV. Attempts to predict the angular distributions have been even less successful and have been attempted only for hydrogen and helium. It is hoped that data such as those presented in this paper will be valuable not only in track structure models but also as experimental results against which some of the new developments in atomic collision theory can be compared. 


\section{FIGURE CAPTIONS}

Figure 1 Schematic drawing of apparatus.

Figure. 2 Cross section for ejection of electrons from hydrogen.

Figure 3 Angular dependence of Energy Transfer in $\mathrm{H}_{2}$.

Figure 4 Angular dependence of energy transfer to ejected electrons having energy $>\varepsilon$ for $1.5 \mathrm{MeV} \cdot \mathrm{H}^{+}$on $\mathrm{H}_{2}$.

Figure 5 Angular dependence of energy transferred to ejected electrons having energy $>\varepsilon$ for $1.7 \mathrm{MeV} \mathrm{H}{ }^{+}$on $\mathrm{N}_{2}$.

Figure 6 Contribution of K-Auger electrons in $\mathrm{N}_{2}$.

Figure 7 Angular dependence of energy transferred to ejected electrons having energy $>\varepsilon$ for $2.0 \mathrm{MeV} \mathrm{H}^{+}$on $\mathrm{Xe}$.

Figure 8 Change in angular dependence of energy transferred to electrons with different proton energies. 


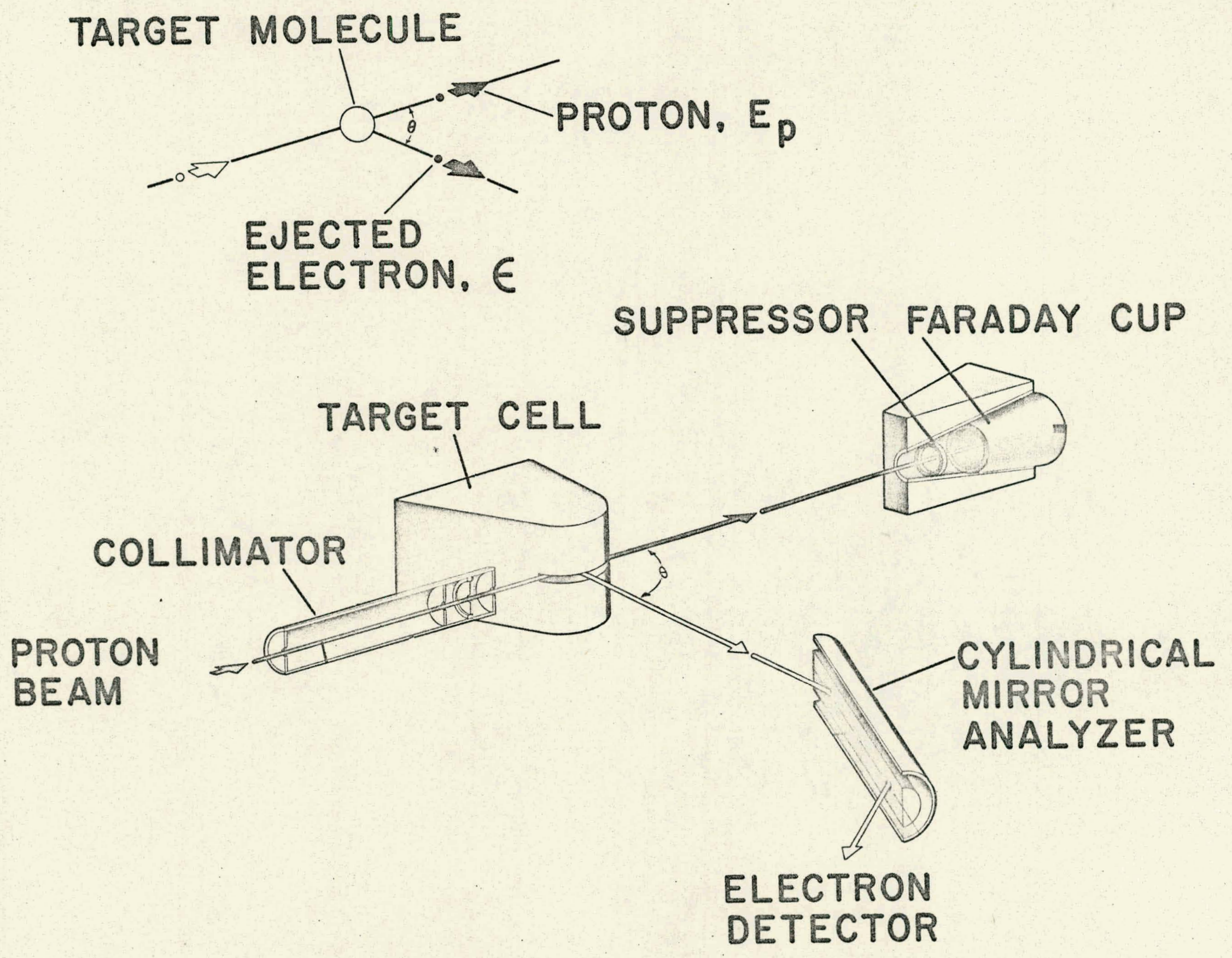




\section{ANGULAR DEPENDENCE OF ENERGY TRANSFER IN $\mathrm{H}_{2}$}

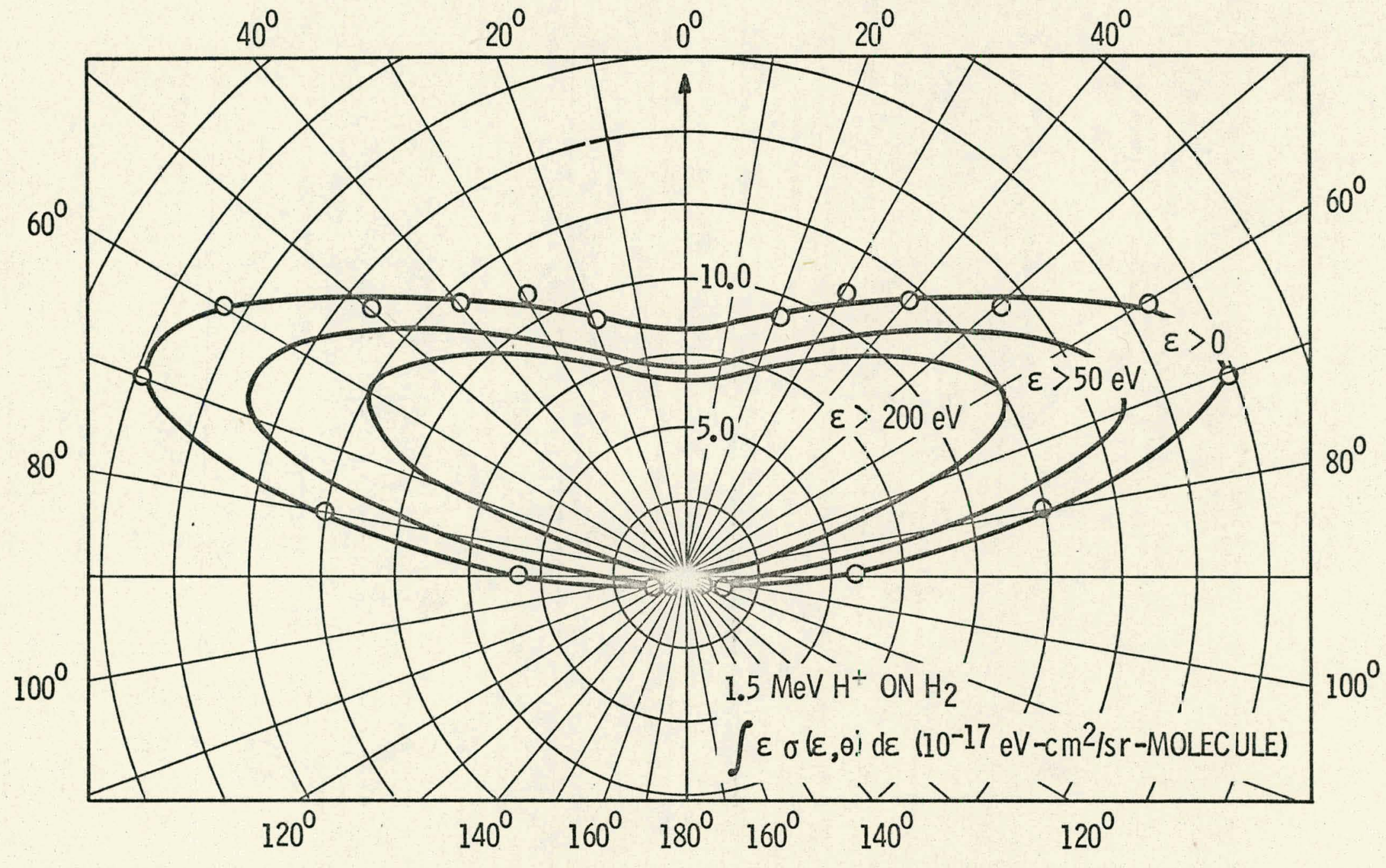




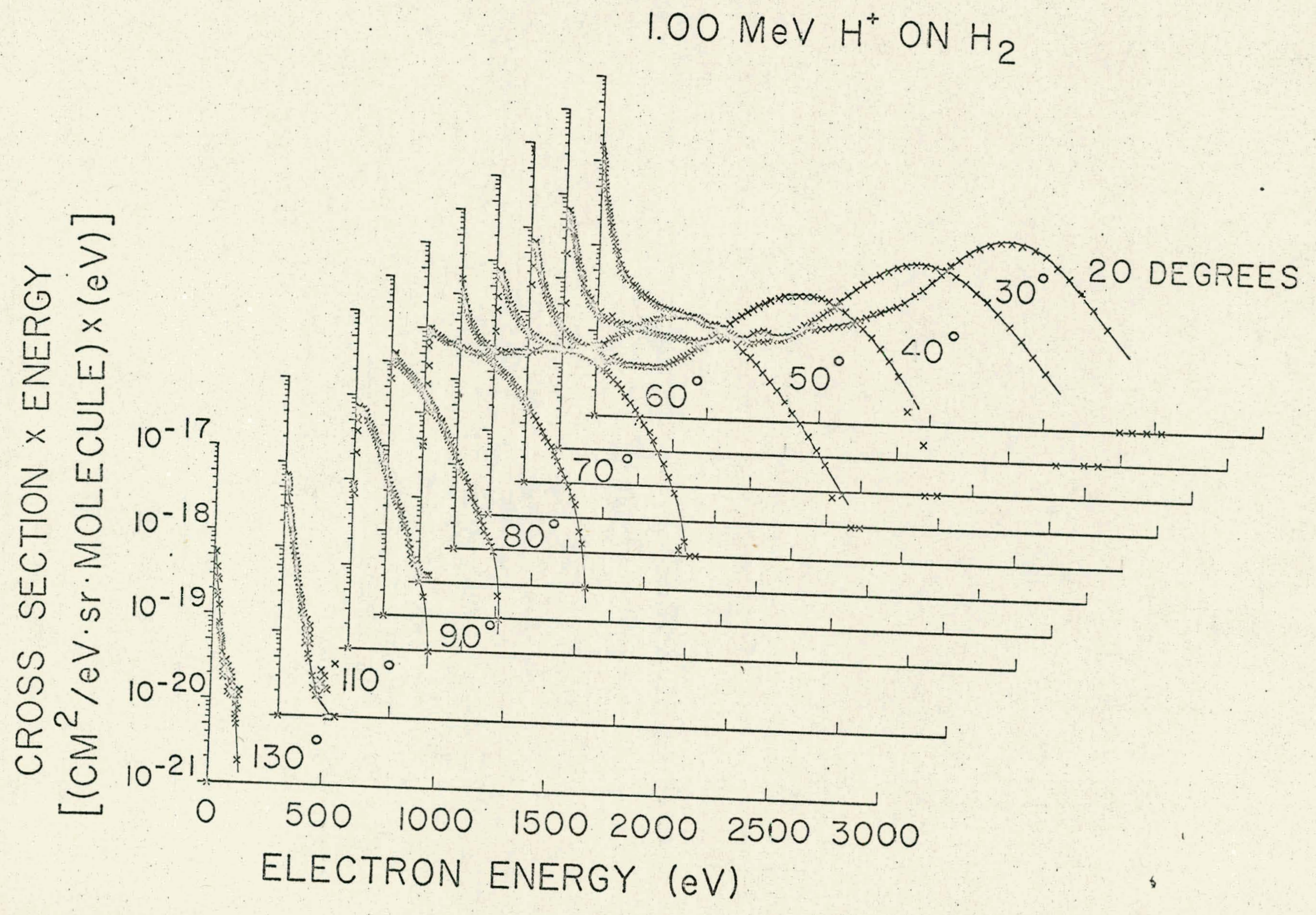

Figueg. 


\section{ANGULAR DEPENDENCE OF ENERGY TRANSFERED TO EJECTED ELECTRONS HAVING ENERGY $>\epsilon$ FOR $1.5 \mathrm{Mev} \mathrm{H}^{+} \mathrm{ON} \mathrm{H}_{2}$}
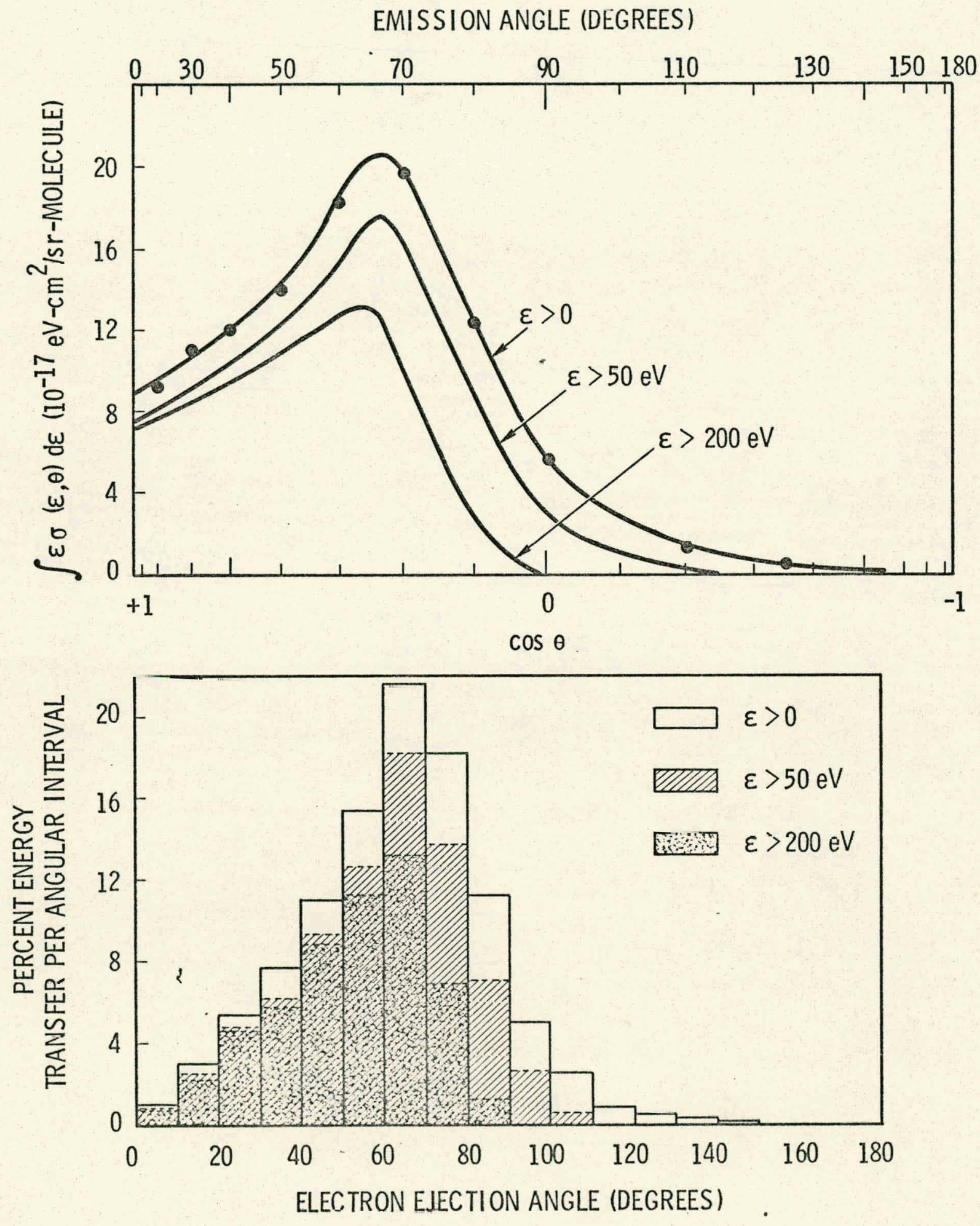


\section{ANGULAR DEPENDENCE OF ENERGY TRANSFERED TO \\ EJECTED ELECTRONS HAVING ENERGY $>\epsilon$ FOR $1.7 \mathrm{MeV} \mathrm{H+ON} \mathrm{N}_{2}$}

- EMISSION ANGLE (DEGREES)
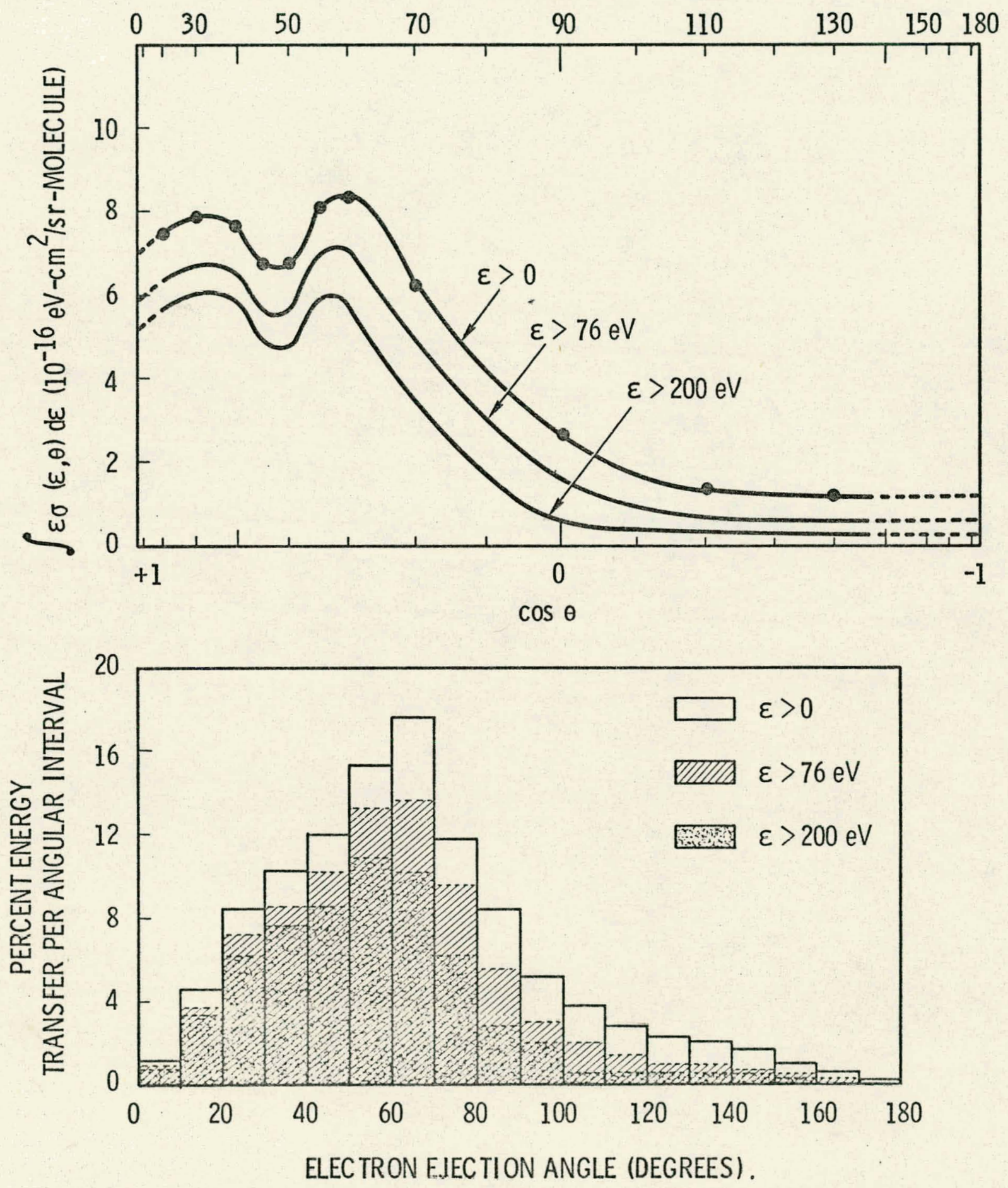


\section{CONTRIBUTION OF K-AUGER ELECTRONS IN $\mathrm{N}_{2}$}

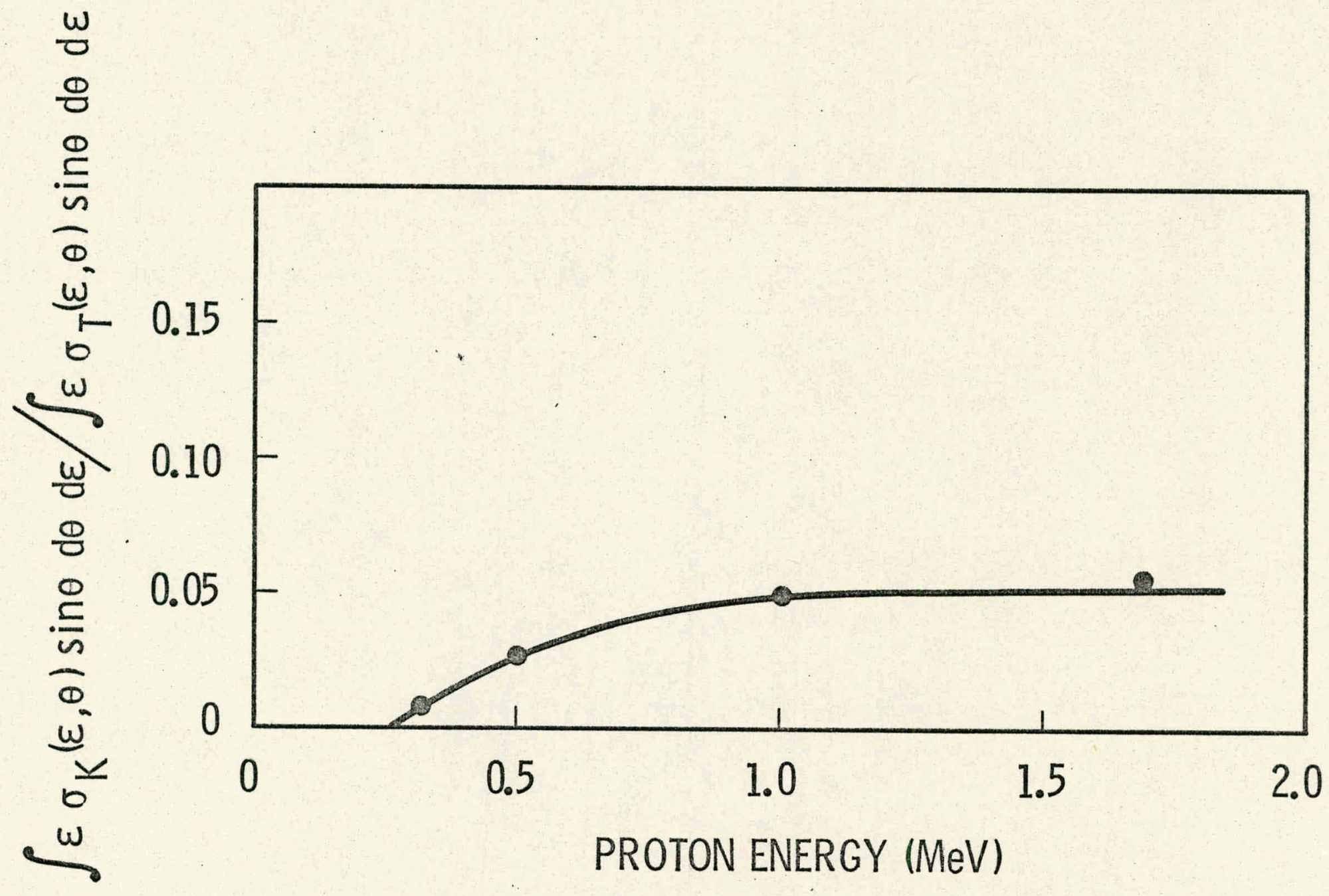




\section{ANGULAR DEPENDENCE OF ENERGY TRANSFERED TO EJECTED ELECTRONS HAVING ENERGY $>\epsilon$ FOR $2.0 \mathrm{Mev} \mathrm{H+ON} \mathrm{Xe}$}

EMISSION ANGLE (DEGREES)
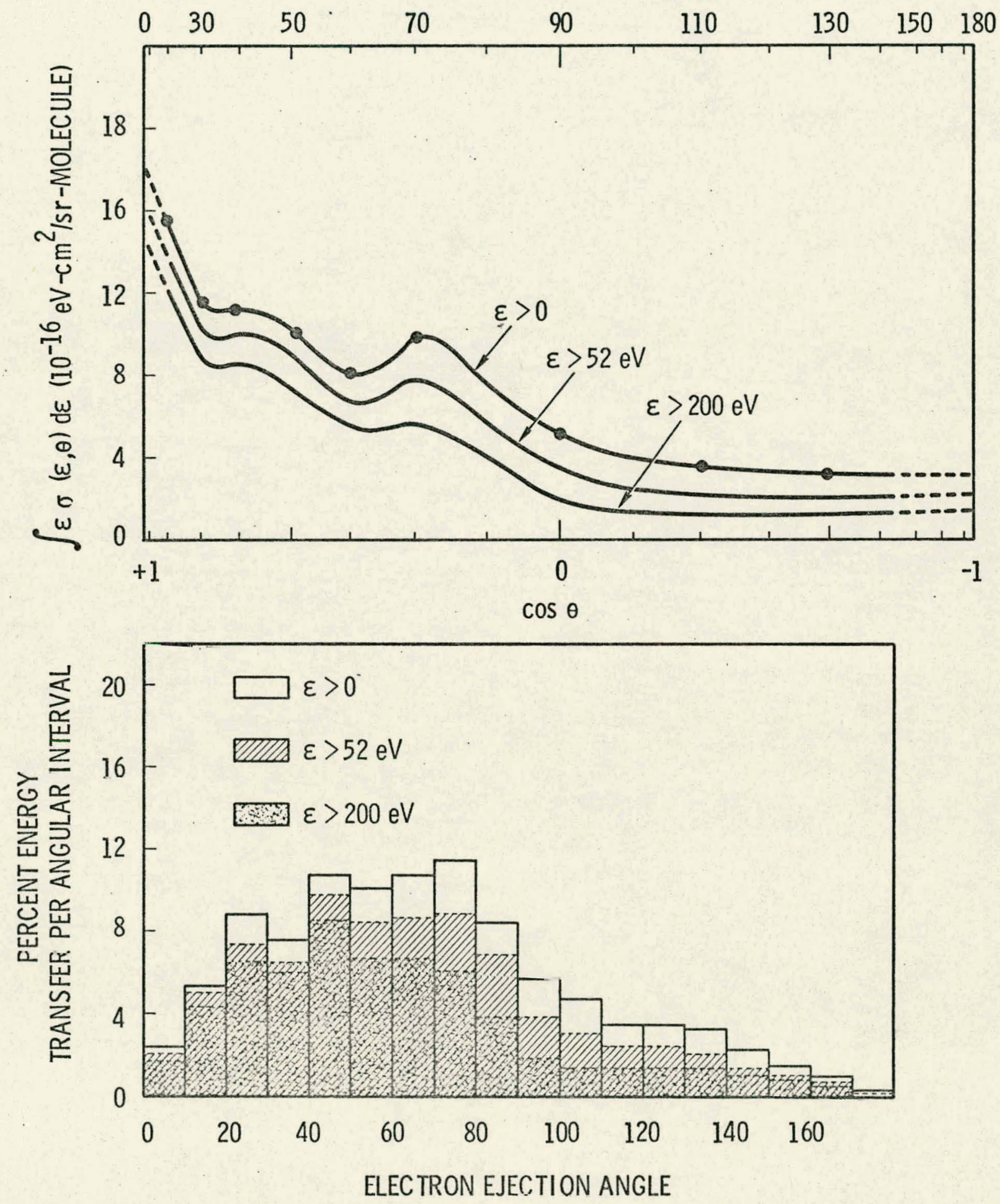
CHANGE IN ANGULAR DEPENDENCE OF ENERGY TRANSFERED TO

ELECTRONS WITH DIFFERENT PROTON ENERGIES

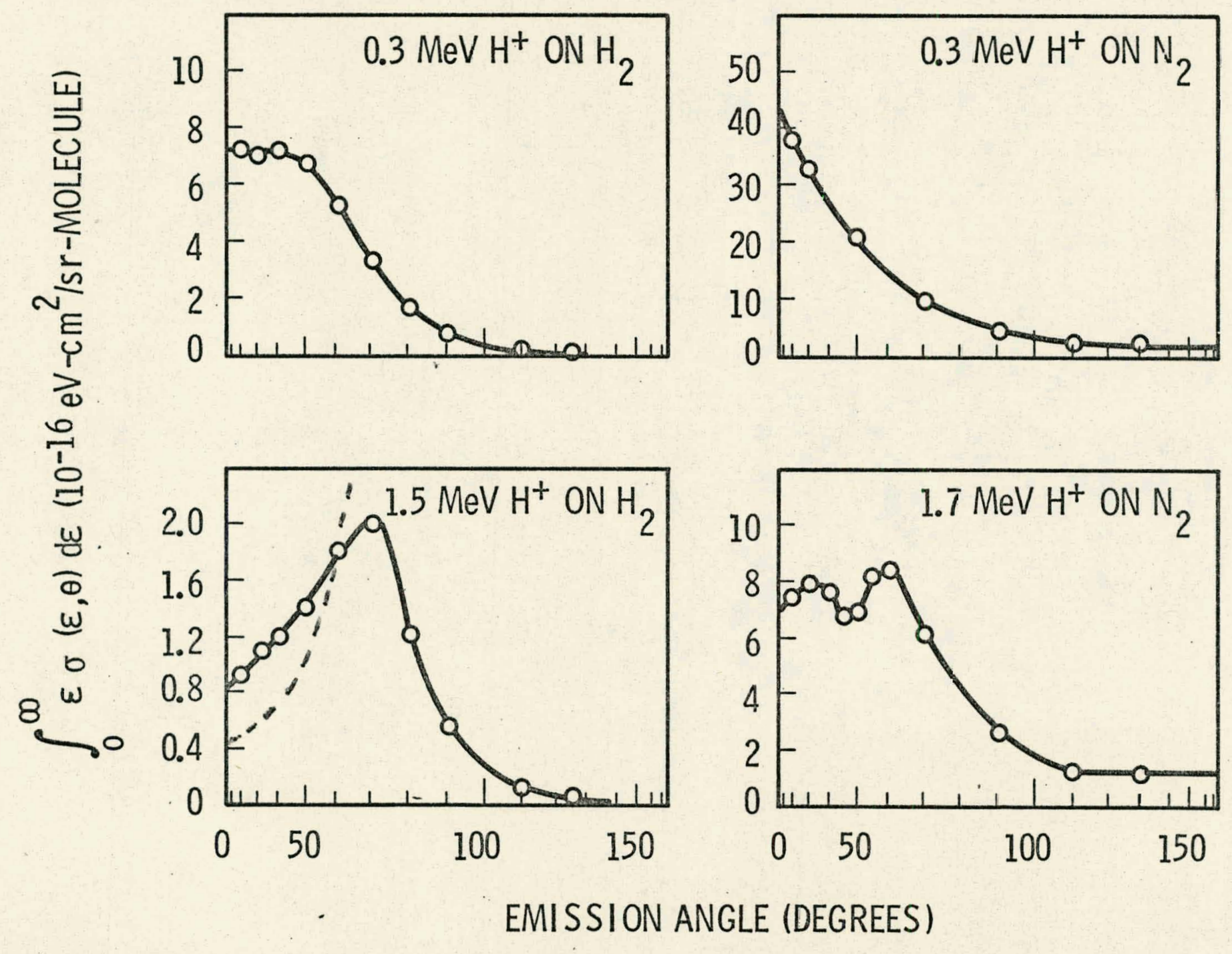

